\title{
Sintomas depressivos em adolescentes em situação de vulnerabilidade social
}

\author{
Depressive symptoms in adolescents in situation of social \\ vulnerability
}

\author{
Diego Grasel Barbosa¹, Rubian Diego Andrade¹, Clarissa Stefani Teixeira², \\ Manoel Gomes Filho Neto ${ }^{1}$, Érico Pereira Gomes Felden ${ }^{1}$
}

\begin{abstract}
Resumo
Objetivo: Identificar os principais indicadores de depressão de amostra de adolescentes em vulnerabilidade social e associar com variáveis sociodemográficas e comportamentais. Método: A amostra foi composta por 135 adolescentes em vulnerabilidade social de Florianópolis, Santa Catarina. Indicadores de depressão foram investigados por meio do Children's Depression Inventory (CDI). Para comparação entre os sexos, utilizou-se o teste $U$ de Mann-Whitney. Com o objetivo de identificar os fatores de agrupamento entre os sintomas depressivos, recorreu-se à análise fatorial com rotação ortogonal varimax normalized. Além disso, a Regressão de Poisson foi utilizada no sentido de identificar as razões de prevalências, tendo como variável desfecho o tercil superior da pontuação do CDI. Resultados: Os indicadores "perda ou ganho de peso", "baixa autoestima" e "fadiga" apresentaram maior poder explicativo dos escores do CDI. Os meninos atingiram maior pontuação média (24,9 pontos). Os índices do CDI foram altos independentemente de questões sociodemográficas, como etnia, e comportamentais, tempo em frente à televisão e ao computador ou videogame. Conclusão: Foram observadas altas prevalências de sintomatologia depressiva nos adolescentes em situação de vulnerabilidade social, com destaque para os meninos, que possuíram 2,24 vezes maior probabilidade de apresentar pontuação elevada no CDI em comparação às meninas.
\end{abstract}

Palavras-chave: depressão; vulnerabilidade social; estilo de vida sedentário; adolescentes; saúde do adolescente.

\begin{abstract}
Objective: To identify the main indicators of depression in a sample of adolescents living under social vulnerability conditions and associate it with sociodemographic and behavioral variables. Method: The sample was composed 135 adolescents in social vulnerability in Florianopolis, Santa Catarina state, Brazil. Indicators of depression were investigated by the Children's Depression Inventory (CDI). The Mann-Whitney test was used for comparison between genders, whereas factor analysis with varimax normalized orthogonal rotation was used to identify grouping factors between depressive symptoms. In addition, Poisson regression was used to identify the prevalence ratios, with the top third of CDI score as the outcome variable. Results: The indicators "weight loss or gain," "low self-esteem", and "fatigue" had greater explanatory power among the CDI scores. Males reached a higher average score (24.9 points) compared with females (23.6 points). The CDI scores were high regardless of sociodemographic and behavioral issues such as ethnicity and time in front of the television, computer, or video game. Conclusion: A high prevalence of depressive symptoms was observed in adolescents in situations of social vulnerability, and males are 2.24 times more likely to achieve high scores on the CDI compared with females.
\end{abstract}

Keywords: depression; social vulnerability; sedentary lifestyle; adolescent; adolescent health.

\footnotetext{
${ }^{1}$ Centro de Ciências da Saúde e do Esporte, Universidade do Estado de Santa Catarina (UDESC) - Florianópolis (SC), Brasil.

${ }^{2}$ Centro Tecnológico, Universidade Federal de Santa Catarina (UFSC) - Florianópolis (SC), Brasil.

Trabalho realizado na Universidade do Estado de Santa Catarina (UDESC) - Florianópolis (SC), Brasil.

Endereço para correspondência: Érico Pereira Gomes Felden - Rua Pascoal Simone, 358 - Coqueiros - CEP: 88080-350 - Florianópolis (SC), Brasil -

Email: ericofelden@gmail.com

Fonte de financiamento: nenhuma.

Conflito de interesses: nada a declarar.
} 


\section{INTRODUÇÃO}

De acordo com a Organização Mundial de Saúde (OMS), estima-se que 350 milhões de pessoas estejam com depressão no mundo ${ }^{1}$. A depressão pode ser caracterizada por transtorno de humor grave, com prejuízo à função mental e com distorção da forma como a pessoa vivencia e entende a realidade. Ocorre em todas as faixas etárias, com um aumento de casos entre jovens e idosos ${ }^{2}$. No entanto, a partir da década de 1970, foi reconhecida em crianças e adolescentes como uma problemática na psiquiatria e, atualmente, é considerada um grave problema de saúde pública ${ }^{3}$.

Considera-se que os sintomas depressivos na infância e na adolescência apresentam natureza duradoura e pervasiva, afetando múltiplas funções e causando significativos danos psicossociais. Adolescentes deprimidos apresentam-se irritáveis e instáveis, podendo ocorrer crises de explosão e raiva em seu comportamento ${ }^{3}$. Entre os 13 e 15 anos, os sintomas depressivos ocorrem na proporção de duas meninas para um menino, e essa diferença tende a permanecer estável durante a vida adulta ${ }^{4}$. Nessa fase da vida, alguns estudos associaram os sintomas de depressão com abusos sofridos na infância, diminuição da concentração de serotonina na fenda pré-sináptica e uma pré-disposição genética ${ }^{1,5}$. Além disso, outras questões de saúde podem estar associadas com os sintomas depressivos, tais como: a autoestima, a satisfação em relação à massa corporal, a satisfação com o corpo, os problemas no sono, o humor deprimido, o baixo interesse e os sentimentos de desesperança ${ }^{6}$. $O$ adolescente pode estar propenso aos sintomas depressivos em decorrência do seu desenvolvimento incompleto, o que não lhe garante a condição de ser autônomo nas suas escolhas e atitudes ${ }^{7}$.

A perspectiva bioética reconhece como vulnerável toda pessoa que se encontra menos apta a se proteger ${ }^{8}$. Vulnerabilidade também pode ser entendida sob o ponto de vista social. De acordo com Malvasi ${ }^{9}$, a vulnerabilidade social em crianças e adolescentes está relacionada a aspectos negativos, principalmente os associados ao envolvimento com drogas, perda de garantia dos direitos e oportunidades nas áreas de educação, saúde e proteção social, com situações de violência, seja ela doméstica ou comunitária, e trabalho infantil. Portanto, caracteriza-se como um conceito multifacetado relacionado aos fatores socioeconômicos e demográficos que afetam a resiliência das comunidades e indivíduos ${ }^{10}$.

Nesse sentido, os acometimentos mentais, como a depressão, podem encontrar-se associados a fatores da vulnerabilidade social ${ }^{11}$. Segundo Fernandes e Milani ${ }^{12}$, o entendimento dos sintomas depressivos na infância é de vital importância para o planejamento do tratamento adequado, de forma que sejam minimizados os riscos ao desenvolvimento da criança em consequência da patologia.

Diante disso, o presente estudo buscou identificar os principais indicadores de depressão do Children's Depression
Inventory ${ }^{13}(\mathrm{CDI})$ em amostra de adolescentes em vulnerabilidade social, bem como associar com variáveis sociodemográficas e comportamentais, visto as crescentes prevalências de depressão na população mundial, o risco aumentado para doenças psíquicas das pessoas em situação de vulnerabilidade social e a escassez de estudos que analisem indicadores de depressão em adolescentes nessa situação.

\section{MÉTODO}

Este estudo caracteriza-se como descritivo com delineamento transversal ${ }^{14}$. A população-alvo foi constituída por adolescentes (10 a 17 anos de idade), moradores de uma região de alta vulnerabilidade social de Florianópolis, Santa Catarina. A amostra foi composta por 135 adolescentes (61\% do sexo masculino) que participam de um projeto de caráter socioeducativo, no qual são realizadas atividades culturais, esportivas, de lazer e tecnologia digital no contraturno escolar. Antes de o adolescente entrar no projeto, ele é avaliado por uma equipe multidisciplinar com o objetivo de identificar a condição de vulnerabilidade social. Entende-se por vulnerabilidade social os adolescentes em situação de risco por negligência (familiar ou do responsável), violência física, psicológica, ou sexual, condições de moradia (lugar perigoso, tráfico, violência urbana) e por contato com dependentes químicos (pais, responsáveis ou pessoas próximas de seu convívio). O projeto atende cerca de 200 jovens ao todo. A amostra foi formada pelos adolescentes que aceitaram participar do estudo e que estavam presente no projeto nos dias da coleta.

Foram investigadas informações por meio de um questionário composto por:

a) dados referentes à identificação do adolescente como idade, sexo e etnia;

b) comportamentos sedentários como tempo em frente à televisão e ao computador ou videogame por dia;

c) depressão infantil, por meio do Children's Depression Inventory (CDI) elaborado por Kovacs ${ }^{13}$, adaptado do inventário BDI (Beck Depression Inventory) para adultos. Esse inventário tem por objetivo identificar alterações afetivas, de humor, de funções autoavaliativas, condutas e ideação suicida. $\mathrm{O}$ instrumento possui 27 itens autoaplicáveis para pessoas de 7 a 17 anos de idade. Para cada questão, existem três opções de resposta $(a=0, b=1, c=2)$, que são somadas ao final, remetendo a uma pontuação máxima de 54 pontos. O participante deve assinalar a resposta considerando o que melhor descreve seu estado nas últimas duas semanas. $\mathrm{O}$ instrumento apresenta coeficiente de consistência interna de 0,81 . No geral são utilizados como pontos de corte 17 pontos em amostras brasileiras ${ }^{15}$. As questões são analisadas e agrupadas nos seguintes indicadores: "humor deprimido", "humor irritável", "baixo interesse", "perda ou ganho de peso", "problemas no sono", "baixa autoestima", "sentimento de desesperança", "fadiga", "falta de atenção/indecisão", "ideação suicida", "comportamento opositivo" e "somatização". 


\section{Procedimentos gerais e questões éticas}

O estudo foi analisado e aprovado pelo Comitê de Ética em Pesquisas com Seres Humanos da Universidade do Estado de Santa Catarina (UDESC), sob parecer n ${ }^{\circ} 37440 / 2013$, e está de acordo com a Resolução no 466/12 do Conselho Nacional de Saúde, que regulamenta as pesquisas envolvendo seres humanos.

Todos os participantes assinaram o termo de assentimento à participação na pesquisa, e o termo de consentimento livre e esclarecido foi assinado pelos pais e/ou responsáveis. As avaliações foram realizadas no local em que são realizadas as atividades do projeto por pesquisadores treinados, e o questionário foi aplicado de forma individual com cada adolescente.

\section{Análise estatística}

Foram realizadas análises descritivas de tendência central e dispersão (médias, desvios-padrões e percentuais). As associações entre as variáveis foram verificadas por meio do qui-quadrado. O teste de U de Mann-Whitney foi empregado para verificar as diferenças entre os grupos.

Para identificar os principais indicadores de depressão infantil na amostra, realizou-se a análise fatorial com vistas a formar agrupamentos relacionados com os 12 indicadores do CDI, considerando fatores com autovalores maiores que 1 . Com o objetivo de minimizar a relação entre os fatores, foi utilizada a rotação ortogonal varimax normalized no cálculo. Nesses fatores, foram consideradas significativas aquelas questões que apresentaram o módulo de $\mathrm{p}>0,7$.

Para identificar as razões de prevalência de sintomas depressivos na amostra analisadas, recorreu-se à Regressão de Poisson, considerando como variável dependente o terceiro tercil da pontuação do CDI (acima de 27 pontos). Foi utilizado o programa estatístico SPSS 20.0 e adotado em todas as análises nível de significância de 5\%.

\section{RESULTADOS}

Os dados descritivos da amostra são apresentados na Tabela 1. A média de idade dos adolescentes investigados foi de 12,8 (1,4) anos e semelhante entre meninos e meninas $(\mathrm{p}=0,417)$. A prevalência do excesso de peso foi de $38 \%$ e não apresentou associação entre os grupos $(\mathrm{p}=0,623)$. A maior parte dos adolescentes se autoclassificou com relação à sua cor da pele como brancos/pardos (57,5\%) ou negros/mulatos (42,5\%), O status de peso não apresentou associação entre os sexos $(\mathrm{p}=0,788)$. Com relação às variáveis comportamentais, $\mathrm{o}$ tempo de utilização de computador ou videogame foi superior no sexo masculino $(\mathrm{p}=0,002)$. Já o tempo em frente à televisão foi maior entre as meninas $(\mathrm{p}=0,854)$.

Na Tabela 2, são apresentadas as pontuações dos 12 indicadores do CDI, com as respectivas médias (desvios-padrõesão) de meninos e meninas. Constatou-se que houve diferenças significativas entre os indicadores "humor irritável" $(p<0,001)$ e "fadiga" ( $p=0,025)$, e em ambos os meninos apresentaram valores superiores.

A pontuação média do somatório do CDI considerando toda a amostra foi $24,4(4,3)$ pontos. Foram observados elevados índices também quando estratificado por sexo ( $\mathrm{p}=0,076)$. Os meninos apresentaram pontuação média de $24,9(4,2)$, e as meninas, 23,6 $(4,3)$ pontos.

Considerando os indicadores do CDI, a "falta de atenção/indecisão" apresentou maiores diferenças com relação ao tempo em

Tabela 1. Dados descritivos da amostra

\begin{tabular}{|c|c|c|c|c|c|c|c|}
\hline \multirow{2}{*}{ Variáveis } & \multicolumn{2}{|c|}{ Total } & \multicolumn{2}{|c|}{ Meninas } & \multicolumn{2}{|c|}{ Meninos } & \multirow{2}{*}{ Valor $\mathbf{p}^{\star *}$} \\
\hline & $\mathbf{n}^{*}$ & $\%$ & $\mathbf{n}^{*}$ & $\%$ & $\mathbf{n}^{\star}$ & $\%$ & \\
\hline Total (\%) & 135 & 100,0 & 53 & 39,0 & 82 & 61,0 & 0,020 \\
\hline \multicolumn{8}{|l|}{ Faixa etária (\%) } \\
\hline 10-12 anos & 58 & 43 & 19 & 35,8 & 39 & 47,6 & 0,179 \\
\hline 13-17 anos & 77 & 57 & 34 & 64,2 & 43 & 52,4 & \\
\hline \multicolumn{8}{|l|}{ Cor da pele (\%) } \\
\hline Brancos/pardos & 65 & 57,5 & 26 & 59,1 & 39 & 56,5 & 0,788 \\
\hline Negros/mulatos & 48 & 42,5 & 18 & 40,9 & 30 & 43,5 & \\
\hline \multicolumn{8}{|l|}{ Status de peso (\%) } \\
\hline Eutróficos & 75 & 66,4 & 28 & 63,6 & 47 & 68,1 & 0,623 \\
\hline Excesso de peso & 38 & 33,6 & 16 & 36,4 & 22 & 31,9 & \\
\hline \multicolumn{8}{|c|}{ Tempo em frente à televisão por dia (\%) } \\
\hline Até 3 horas & 66 & 57,9 & 25 & 56,8 & 41 & 58,6 & 0,854 \\
\hline 4 ou mais horas & 48 & 42,1 & 19 & 43,2 & 29 & 41,4 & \\
\hline \multicolumn{8}{|c|}{$\begin{array}{l}\text { Tempo no computador ou videogame } \\
\text { por dia }(\%)\end{array}$} \\
\hline Até 2 horas & 70 & 61,4 & 35 & 79,5 & 35 & 50,0 & 0,002 \\
\hline 3 ou mais horas & 44 & 38,6 & 9 & 20,5 & 35 & 50,0 & \\
\hline
\end{tabular}

${ }^{*}$ Missings existentes em algumas variáveis por não resposta. ${ }^{* \star}$ Teste de qui-quadrado 
frente à televisão $(\mathrm{p}=0,038)$ (Figura 1$)$. Esse indicador inclui questões como a capacidade do adolescente tomar decisão, capacidade de concentração, ânimo para realizar as tarefas escolares e problemas com as notas da escola. Especificamente com relação a este último item, a frequência de alunos que afirmaram "tenho me saído mal em matérias que costumava ir bem" foi superior no grupo que assistia quatro ou mais horas de TV por dia $(\mathrm{p}=0,025)$.

Outro comportamento importante refere-se ao tempo destinado ao computador e videogame. $\mathrm{O}$ indicador que apresentou maior diferença nesse comportamento foi o "humor irritável" ( $\mathrm{p}=0,043)$ (Figura 1). Esse indicador contempla as questões "eu me sinto sempre preocupado" e "eu estou sempre envolvido em brigas". Esta última apresentou maior diferença entre os que passam maior tempo em atividades virtuais $(\mathrm{p}=0,052)$.

Por meio da análise fatorial (Tabela 3), verificou-se que as variáveis "perda ou ganho de peso", "baixa autoestima" e "fadiga" foram as questões com maior poder de explicação da depressão infantil (20,9\%) considerando os indicadores do CDI em amostra de adolescentes em situação de vulnerabilidade social.

$\mathrm{Na}$ Tabela 4, são apresentadas as razões de prevalência utilizando a Regressão de Poisson. Como possíveis pontos de corte do CDI apresentados pela literatura não foram adequados para esta amostra, optou-se por utilizar tercis da distribuição da soma do CDI e considerar o tercil superior como desfecho na análise de Regressão de Poisson. A variável sexo foi a única apresentar associação com o desfecho. Os meninos apresentaram duas vezes maior probabilidade de estarem no tercil superior da pontuação do CDI $(\mathrm{RP}=2,24$; IC95\%=1,1-1,4) em relação às meninas.

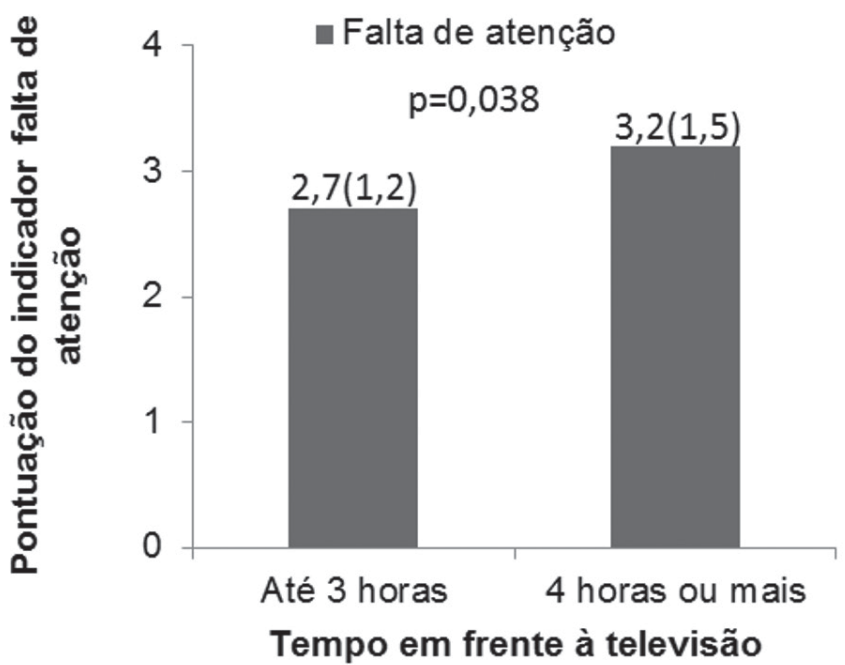

Tabela 2. Médias, desvios-padrão e diferenças entre as médias de meninos e meninas nos indicadores do CDI

\begin{tabular}{lccc}
\multicolumn{1}{c}{ Dimensões do CDI } & \multicolumn{2}{c}{ Média (desvio-padrão) } & p-valor \\
& Meninos & Meninas & p-ver \\
Humor deprimido & $3,8(1,0)$ & $3,6(1,4)$ & 0,296 \\
Humor irritável & $1,9(1,1)$ & $1,0(1,0)$ & $<\mathbf{0 , 0 0 1}$ \\
Baixo interesse & $2,1(1,2)$ & $2,3(1,1)$ & 0,707 \\
Perda ou ganho de peso & $1,5(0,6)$ & $1,2(0,7)$ & $\mathbf{0 , 0 5 1}$ \\
Problemas no sono & $0,6(0,6)$ & $0,7(0,6)$ & 0,195 \\
Baixa autoestima & $6,2(1,6)$ & $5,9(2,0)$ & 0,537 \\
Sentimento de desesperança & $2,7(1,2)$ & $2,3(1,3)$ & 0,117 \\
Fadiga & $1,7(0,6)$ & $1,4(0,7)$ & $\mathbf{0 , 0 2 5}$ \\
Falta de atenção/indecisão & $3,0(1,5)$ & $2,7(1,3)$ & 0,328 \\
Ideação suicida & $0,4(0,6)$ & $0,5(0,6)$ & 0,072 \\
Comportamento opositivo & $0,6(0,7)$ & $0,7(0,7)$ & 0,183 \\
Somatização & $0,7(0,7)$ & $0,8(0,6)$ & 0,332 \\
\hline
\end{tabular}

*Teste $U$ de Mann-Whitney

Tabela 3. Distribuição das questões do CDI de acordo com os fatores de agrupamento

\begin{tabular}{|c|c|c|c|}
\hline Fatores & Questões & $\mathbf{r}$ & $\begin{array}{c}\text { Explicação } \\
(\%)\end{array}$ \\
\hline \multirow{3}{*}{1} & Baixa autoestima & 0,876 & \multirow{3}{*}{20,9} \\
\hline & Fadiga & 0,738 & \\
\hline & Perda ou ganho de peso & 0,700 & \\
\hline \multirow{2}{*}{2} & Humor deprimido & 0,768 & \multirow{2}{*}{12,6} \\
\hline & Falta de atenção & 0,628 & \\
\hline 3 & $\begin{array}{l}\text { Sentimento de } \\
\text { desesperança } \\
\text { Problemas com o sono }\end{array}$ & $\begin{array}{l}0,880 \\
0,782\end{array}$ & 11,0 \\
\hline 4 & Baixo interesse & 0,775 & 9,9 \\
\hline 5 & $\begin{array}{l}\text { Comportamento } \\
\text { opositivo }\end{array}$ & 0,747 & 8,9 \\
\hline
\end{tabular}

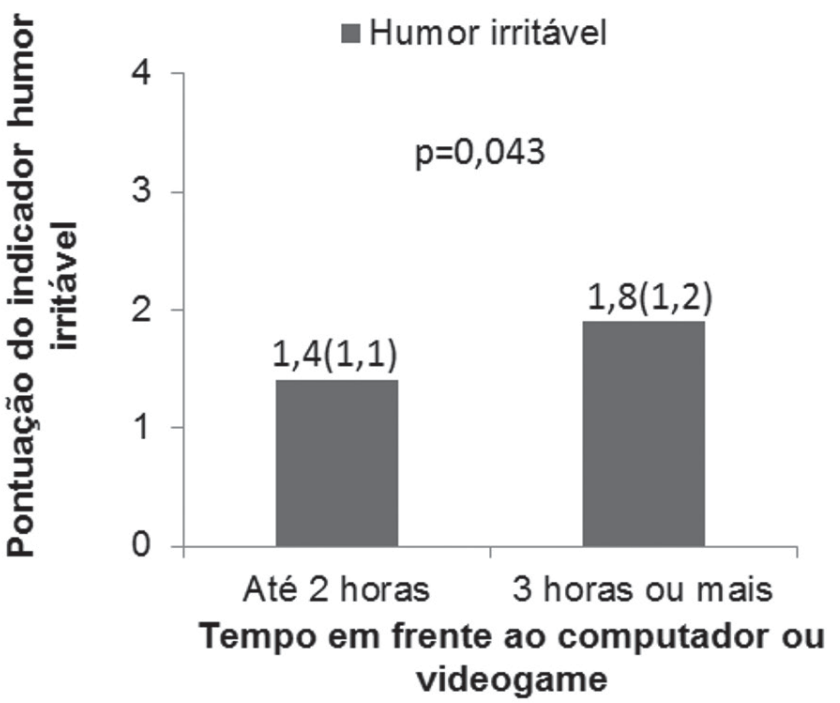

Figura 1. Diferença entre a falta de atenção e tempo em frente à televisão, e entre humor irritável e tempo em frente ao videogame ou computador 
Tabela 4. Razões de prevalências (RP) de Poisson, utilizando-se como variável dependente o tercil superior $(n=33)$ da pontuação do CDI

\begin{tabular}{|c|c|c|c|}
\hline Variáveis & $\begin{array}{c}\text { Prevalência } \\
(\%)\end{array}$ & RP (IC95\%) & p-valor \\
\hline $\begin{array}{l}\text { Faixa etária } \\
\text { 10-12 anos } \\
13-17 \text { anos }\end{array}$ & $\begin{array}{l}30,2 \\
34,5 \\
\end{array}$ & $\begin{array}{c}1 \\
1,14(0,7-2,0)\end{array}$ & 0,647 \\
\hline $\begin{array}{l}\text { Sexo } \\
\text { Masculino } \\
\text { Feminino } \\
\end{array}$ & $\begin{array}{l}41,3 \\
18,4 \\
\end{array}$ & $\begin{array}{c}2,24(1,1-4,8) \\
1\end{array}$ & $0,018^{*}$ \\
\hline $\begin{array}{l}\text { Cor da pele } \\
\text { Brancos/pardos } \\
\text { Negros/mulatos }\end{array}$ & $\begin{array}{l}32,8 \\
32,6 \\
\end{array}$ & $\begin{array}{c}1,01(0,6-1,6) \\
1 \\
\end{array}$ & 0,873 \\
\hline $\begin{array}{l}\text { Status de peso } \\
\text { Eutróficos } \\
\text { Excesso de peso }\end{array}$ & $\begin{array}{l}39,4 \\
20,6 \\
\end{array}$ & $\begin{array}{c}1,91(0,9-3,9) \\
1\end{array}$ & 0,123 \\
\hline $\begin{array}{l}\text { Tempo em frente à TV } \\
\text { Até } 3 \text { horas } \\
4 \text { ou mais horas }\end{array}$ & $\begin{array}{l}26,2 \\
42,5 \\
\end{array}$ & $\begin{array}{c}1,62(1,0-2,9) \\
1\end{array}$ & 0,060 \\
\hline $\begin{array}{l}\text { Tempo no computador } \\
\text { ou videogame } \\
\text { Até } 2 \text { horas } \\
3 \text { ou mais horas }\end{array}$ & $\begin{array}{l}33,3 \\
31,6 \\
\end{array}$ & $\begin{array}{c}1,05(0,6-1,8) \\
1 \\
\end{array}$ & 0,222 \\
\hline
\end{tabular}

${ }^{*}$ p-valor $<0,05$

\section{DISCUSSÃO}

O presente estudo buscou descrever os indicadores de depressão do CDI em uma amostra de adolescentes em vulnerabilidade social e associá-los com variáveis sociodemográficas e comportamentais. De forma geral, os jovens em vulnerabilidade social analisados apresentam elevada pontuação para os sintomas depressivos. A avaliação dos sintomas mostrou indicadores de morbidade mais elevados nesta amostra quanto que os apresentados pela literatura.

No estudo de Fonseca et al. ${ }^{16}$, no qual foi investigada a presença de sintomas depressivos de 519 estudantes do interior de Minas Gerais de 7 a 13 anos, foi observada prevalência de $13,7 \%$, considerando o valor de 17 como ponto de corte, conforme sugerido por Gouveia et al. $^{15}$. Se esse mesmo parâmetro fosse utilizado com os adolescentes em vulnerabilidade social analisados neste estudo, a prevalência seria de aproximadamente 98\%. Além disso, o valores mínimos e máximos da escala (14 e 33 pontos, respectivamente) reafirmam que o ponto de corte sugerido pela literatura pode não ser adequado para esta amostra e, por essa razão, os critérios de análise necessitam de aprofundamento.

Alguns fatores são apresentados pela literatura como possíveis potencializadores ou protetores para os sintomas depressivos. Em um estudo, Campos et al. ${ }^{17}$ buscaram investigar quais variáveis sociodemográficas e habilidades sociais podem ser fatores de risco ou proteção da depressão na adolescência. Os autores verificaram que o sexo feminino $(\mathrm{OR}=4,22 ; \mathrm{IC} 95 \%=2,5-7,1)$ e o fator civilidade $(\mathrm{OR}=1,10 ; \mathrm{IC} 95 \%=1,0-1,2)$ são fatores de risco, e a variável idade de 12 anos $(\mathrm{OR}=0,46 ; \mathrm{IC} 95 \%=0,2-0,8)$, habilidades de empatia $(\mathrm{OR}=0,97$; IC95\%=0,9-1,0) e o autocontrole $(\mathrm{OR}=0,940$; IC95\%=0,904-0,978) são fatores de proteção à depressão.

Como se sabe, a depressão costuma ser mais frequente no sexo feminino ${ }^{4}$, porém, neste estudo, a prevalência maior foi no sexo oposto. Supõe-se que tal fato esteja associado às características desse grupo em vulnerabilidade social, bem como ao possível risco de associação com uso de álcool e outras drogas ${ }^{18}$. Em estudo de Aglio e Hutz ${ }^{19}$, em que investigaram questões de depressão infantil e rendimento escolar de 215 crianças e adolescentes de 7 a 15 anos, de escolas da periferia de Porto Alegre, Rio Grande do Sul, foi verificada média de pontuação dos indicadores do CDI maior nas meninas (15,8 pontos) quando comparados aos meninos (13,6 pontos). Os autores explicam tal situação considerando maior frequência de violência doméstica e abuso sexual sofrida pelas meninas. No presente estudo, porém, foram observadas maiores pontuações dos indicadores do CDI nos meninos ( 24,9 pontos) do que nas meninas ( 23,6 pontos) $(\mathrm{p}=0,076)$. Além disso, os meninos apresentaram 2,24 vezes mais probabilidade de apresentar sintomatologia depressiva quando comparados ao sexo oposto $(\mathrm{RP}=2,24$; IC95\%=1,1-1,4). Tal situação deve ser aprofundada em futuras investigações com adolescentes em situação de vulnerabilidade social, considerando que os meninos, possivelmente, estejam mais suscetíveis ao envolvimento com tráfico de drogas e situações de violência na rua, bem como consumo de álcool.

No presente estudo, diferenças significativas foram identificadas entre os sexos nos indicadores "humor irritável" e "fadiga" do CDI. No que diz respeito ao humor irritável, Stringaris et al. ${ }^{20}$ identificaram que a depressão, quando analisada como um somatório de sintomas, foi maior nas meninas, porém, quando a depressão e o humor irritável foram analisados em conjunto, os meninos apresentaram os maiores valores. Isso indica que os meninos tendem a apresentar maiores comportamentos de humor irritável e agressividade associados à depressão em comparação às meninas. Dessa forma, mesmo que as diferenças individuais nos tratamentos e intervenções sejam fundamentais, as medidas para o controle e diminuição de sintomas depressivos podem ser diferentes de acordo com o sexo.

Além dos fatores já apresentados, estudos recentes vêm revelando associação entre a presença de problemas psíquicos e agressividade com a dependência do uso da internet e jogos eletrônicos por adolescentes. Esse efeito pode ser mais bem entendido por meio dos processos maturacionais do cérebro durante essa fase. Hahn e Klin ${ }^{21}$ sugerem que, tanto áreas do córtex pré-frontal e do sistema límbico, como neurotransmissores (dopamina, noradrenalina e serotonina), estão associadas com os dois fenômenos. Assim, a utilização frequente da internet 
e de jogos eletrônicos pode ser um fator de risco para tal comportamento, especialmente nos adolescentes que estão em processo de desenvolvimento do córtex pré-frontal, responsável pelo controle de condutas violentas, e nos meninos que parecem ser mais vulneráveis a desenvolver hábitos problemáticos na prática de jogos eletrônicos ${ }^{22}$. No presente estudo, os meninos apresentaram maior quantidade de tempo na utilização de computador ou videogame, e isso pode ter influenciado indiretamente na maior pontuação da depressão infantil, pois se supõe que o humor irritável possa ter relação com o conteúdo violento dos jogos de computador e/ou videogame.

O percentual de adolescentes que assistiam quatro ou mais horas de TV por dia foi de 42,1\%. Nesse sentido, é provável que os adolescentes, ao ficarem longos períodos de tempo em frente à TV, deixem de realizar atividades ao ar livre, como jogos, esportes e passeios. Por essa razão, é recomendado que crianças e adolescentes assistam não mais que uma a duas horas de TV com programação de qualidade ${ }^{22}$. Uma das consequências mais imediatas desses comportamentos pode ser identificada pelo aumento das prevalências de sobrepeso e obesidade, como analisado no estudo de Rivera et al. ${ }^{23}$, no qual identificou associação entre obesidade e assistir três horas ou mais de televisão por dia.

No sentido de compreender as causas dos sintomas depressivos dos adolescentes em vulnerabilidade social, as questões com maior poder de explicação foram: "baixa autoestima", "fadiga" e "perda ou ganho de peso". Essas questões interligadas entre si ajudaram a explicar um quinto da distribuição dos escores do CDI. No que se refere à baixa autoestima e sua relação com sintomas depressivos na adolescência, estudo longitudinal, com 674 adolescentes mexicanos de 10 e 12 anos $^{23}$, concluiu que a presença de baixa autoestima aos 10 anos foi preditora de depressão aos 12 anos. Além disso, a fadiga associada à baixa autoestima e à perda ou ganho de peso também teve influência no aumento de sintomas depressivos nos adolescentes.

Diante disso, como sugere Abelha ${ }^{24}$, promover atitudes positivas da comunidade para com as pessoas com problemas psíquicos e estimular a procura pelo tratamento são atitudes urgentes da saúde pública. Assim, se a depressão foi significativamente identificada em um grupo de adolescentes em vulnerabilidade social, poderíamos inferir que ações de melhorias das condições sociais poderiam contribuir para a diminuição das prevalências de depressão. Portanto, as ações para desenvolvimento humano devem ser estimuladas, ou seja, melhorias na educação, condições de moradia, trabalho, renda e saúde, além de ações específicas considerando a avaliação e o acompanhamento visando à melhoria da autoestima, controle do peso corporal e horários de descanso e repouso.

Aponta-se como principal limitação deste estudo a impossibilidade de analisar relações de causa e efeito. Além disso, um número superior de meninas poderia fortalecer as análises. No entanto, o presente estudo levanta discussões importantes à comunidade científica acerca dessa população carente de atenção e pesquisa. Novos estudos relacionados à depressão infantil, inclusive de acompanhamento, devem ser fomentados, uma vez que essa patologia é um grande problema de saúde pública. Sugere-se como pesquisas futuras investigar a relação do tipo de jogo e/ou qualidade dos programas de televisão com a depressão infantil.

\section{CONCLUSÃO}

Foram observadas altas prevalências de sintomatologia depressiva nos adolescentes em situação de vulnerabilidade social. Verificou-se que as variáveis "perda ou ganho de peso", "baixa autoestima" e "fadiga" explicaram 20,9\% da depressão infantil de adolescentes em situação de vulnerabilidade social. Além disso, os meninos possuem 2,24 vezes maior probabilidade de apresentar pontuação elevada no CDI em comparação às meninas.

Contudo, ações de melhorias das condições sociais, como maior acesso à educação, saúde e lazer, poderiam contribuir para a diminuição das prevalências de depressão de adolescentes em situação de vulnerabilidade social.

\section{REFERÊNCIAS}

1. Organización Mundial de la Salud. Informe sobre la salud en el mundo 2001. Salud mental: nuevos conocimientos, nuevas esperanzas. Ginebra: OMS; 2001

2. Miranda MV, Firmo WCA, Castro NG, Alves LPL, Dias CN, Rêgo MM, et al Depressão infantil: aspectos gerais, diagnóstico e tratamento. Cadernos Pesquisa. 2013;20(3)

3. Huttel J, Kisxiner KA, Bonetti RA, Rosa MIPD. A depressão infantil e suas formas de manifestação. Psicol Argum. 2011;29(64):11-22.

4. Hankin BL. Cognitive vulnerability-stress model of depression during adolescence: investigating depressive symptom specificity in a multi-wave prospective study. J Abnorm Child Psychol. 2008;36(7):999-1014. http:// dx.doi.org/10.1007/s10802-008-9228-6. PMid:18437551.

5. Van der Auwera S, Janowitz D, Schulz A, Homuth G, Nauck M, Volzke $\mathrm{H}$, et al. Interaction among childhood trauma and functional polymorphisms in the serotonin pathway moderate the risk of depressive disorders. Eur Arch Psychiatry Clin Neurosci. 2014;264(S1 Supl 1):S45-54. http://dx.doi. org/10.1007/s00406-014-0536-2. PMid:25214390.

6. Rawana J, Morgan A. The relation between eating-and weight-related disturbances and depressive symptoms among early and late adolescents. J Nutr Disorders Ther S. 2011;2:2161-0509.

7. Zappe JG, Santos CR, Ferrão IS, Dias ACG. Vulnerabilidade e autonomia na pesquisa com adolescentes privados de liberdade. Psicologia: Ciência e Profissão. 2013;33(1):234-47.

8. Diniz D, Corrêa M. Declaração de Helsinki: relativismo e vulnerabilidade. Cad Saude Publica. 2001;17(3):679-88. http://dx.doi.org/10.1590/S0102311X2001000300022. PMid:11395804. 
9. Malvasi PA, Adorno RCF. A vulnerabilidade ea mente: conflitos simbólicos entre o diagnóstico institucional ea perspectiva de jovens em cumprimento de medida socioeducativa. Saúde e Sociedade. 2014;23(1):30-41. http:// dx.doi.org/10.1590/S0104-12902014000100002.

10. Flanagan BE, Gregory EW, Hallisey EJ, Heitgerd JL, Lewis B. A social vulnerability index for disaster management. J Homel Secur Emerg Manage. 2011;8(1):1-24.

11. Hovens JG, Giltay EJ, Spinhoven P, Van Hemert AM, Penninx BW. Impact of childhood life events and childhood trauma on the onset and recurrence of depressive and anxiety disorders. J Clin Psychiatry. 2015;76(7):931-8. http://dx.doi.org/10.4088/JCP.14m09135. PMid:25699690.

12. Fernandes AM, Milani RG. A depressão infantil, o rendimento escolar e a autoeficácia: uma revisão da literatura. Revista Cesumar. 2010;15(2):381-403.

13. Kovacs M. The Children's Depression, Inventory (CDI). Psychopharmacol Bull. 1985;21(4):995-8. PMid:4089116.

14. Gil AC. Como elaborar projetos de pesquisa. 5. ed. São Paulo: Atlas; 2010.

15. Gouveia VV, Barbosa GA, Almeida HJF, Gaião AA. Inventário de depressão infantil-CDI: estudo de adaptação com escolares de João Pessoa. J Bras Psiquiatr. 1995;44(7):345-9.

16. Fonseca MHG, Ferreira RA, Fonseca SG. Prevalência de sintomas depressivos em escolares. Pediatria. 2005;27(4):223-32.

17. Campos JR, Del Prette A, Del Prette ZAP. Depressão na adolescência: habilidades sociais e variáveis sociodemográficas como fatores de risco/ proteção. Estud Pesqui Psicol. 2014;14(2):408-28. http://dx.doi.org/10.12957/ epp.2014.12645.
18. Manso DSS, Matos MG. Depressão, ansiedade e consumo de substâncias em adolescentes. Rev Bras Ter Cogn. 2006;2(1):73-84.

19. Aglio DDD, Hutz CS. Depressão e desempenho escolar em crianças e adolescentes institucionalizados. Psicol Reflex Crit. 2004;17(3):341-50.

20. Stringaris A, Maughan B, Copeland WS, Costello EJ, Angold A. Irritable mood as a symptom of depression in youth: prevalence, developmental, and clinical correlates in the great smoky mountains study. J Am Acad Child Adolesc Psychiatry. 2013;52(8):831-40. http://dx.doi.org/10.1016/j. jaac.2013.05.017. PMid:23880493.

21. Hahn C, Kim DJ. Is there a shared neurobiology between aggression and Internet addiction disorder? J Behav Addict. 2014;3(1):12-20. http://dx.doi. org/10.1556/JBA.3.2014.1.2. PMid:25215210.

22. American Academy of Pediatrics. Committee on Public Education. American Academy of Pediatrics. Children, adolescents, and television. Pediatrics. 2001;107(2):423-6. http://dx.doi.org/10.1542/peds.107.2.423. PMid:11158483.

23. Rivera IR, Silva MAM, Silva RDTA, Oliveira ACCC, Carvalho ACC. Atividade física, horas de assistência à TV e composição corporal em crianças e adolescentes. Arq Bras Cardiol. 2010;95(2):159-65. http://dx.doi. org/10.1590/S0066-782X2010005000065. PMid:20563518.

24. Abelha L. Depressão: uma questão de saúde pública. Cad Saúde Colet. 2014;22(3):223.

Recebido em: Set. 09, 2015 Aprovado em: Abr. 06, 2016 